ARTICLE

Received 9 May 2013 | Accepted 9 Oct 2013 | Published 1 Nov 2013 DOl: 10.1038/ncomms3737

\title{
Muscle function and hydrodynamics limit power and speed in swimming frogs
}

\author{
Christofer J. Clemente ${ }^{1} \&$ Christopher Richards ${ }^{2}$
}

Studies of the muscle force-velocity relationship and its derived $n$-shaped power-velocity curve offer important insights into muscular limits of performance. Given the power is maximal at $1 / 3 V_{\max }$ geometric scaling of muscle force coupled with fluid drag force implies that this optimal muscle-shortening velocity for power cannot be maintained across the natural body-size range. Instead, muscle velocity may decrease with increasing body size, conferring a similar $n$-shaped power curve with body size. Here we examine swimming speed and muscle function in the aquatic frog Xenopus laevis. Swimming speed shows an $n$-shaped scaling relationship, peaking at $47.35 \mathrm{~g}$. Further, in vitro muscle function of the ankle extensor plantaris longus also shows an optimal body mass for muscle power output $(47.27 \mathrm{~g}$ ), reflecting that of swimming speed. These findings suggest that in drag-based aquatic systems, muscle-environment interactions vary with body size, limiting both the muscle's potential to produce power and the swimming speed.

\footnotetext{
${ }^{1}$ School of Biological Sciences, University of Queensland, Goddard Building, St Lucia, Brisbane, Queensland 4072, Australia. ${ }^{2}$ Rowland Institute for Science at Harvard, 100 Edwin Land Boulevard, Cambridge, Massachusetts 02142, USA. Correspondence and requests for materials should be addressed to C.J.C. (email: c.clemente@uq.edu.au).
} 
C lassic studies modelling the interplay between animal speed and body size have often predicted a linear relationship between these variables ${ }^{1}$. Yet, the fastest animals are often neither the largest nor the smallest, but are intermediately sized, producing an $n$-shaped curve between speed and body mass ${ }^{2-4}$. More recent studies on terrestrial systems have suggested that this intermediate limit to sprint speed may be based on a reduced ability of limb bones and muscles to support body mass at larger sizes ${ }^{5,6}$. However, muscle power (muscle force $\times$ muscle-shortening velocity), used to propel the body forward, may also limit locomotor ability. Previous studies on terrestrial lizards have reported that muscle power may not limit sprint speed, as absolute muscle power output increases when running on greater inclines or when loaded with weights ${ }^{7,8}$, suggesting muscles are running at a submaximal power when unloaded or on level surfaces. However, the extent to which muscle power may limit speed is unclear, because we cannot easily decouple a muscle's contribution to weight support versus propulsion. To examine muscle power limits to speed, we need to control for or remove support limitations with mass. In this regard, aquatic systems are ideal, as body mass is supported by buoyancy, removing weight support as a speed limitation. This may help determine the extent to which muscle power limits performance.

The aquatic frog, Xenopus laevis, is particularly suitable for such studies, as it powers its kick almost exclusively via ankle rotation as described by Richards ${ }^{9}$. Thus, swimming speed is correlated to plantaris longus (PL) muscle power, which extends the ankle ${ }^{10}$. Similar to all muscles, this muscle is governed by two well-known properties. First, the force generated by muscle fibres depends on the instantaneous length of the sarcomeres, with maximal force occurring when the thick and thin filaments (myosin and actin) are near $100 \%$ overlap $^{11}$. Second, the force generated by muscle fibres decreases hyperbolically as muscle-shortening velocity increases ${ }^{12}$. This second property can also be represented as a power-velocity curve (power $=$ force $\times$ velocity), where power is maximal at $\sim 1 / 3$ of the maximum muscle-shortening velocity $\left(V_{\max }\right)$, but decreases at greater and lesser velocities, producing an ' $n$-shaped' curve. Thus, for the muscle to produce its maximal mechanical power, it must be maximally stimulated, be near $100 \%$ overlap and operate at $\sim 1 / 3 V_{\max }{ }^{11,13}$. However, below we derive a scaling model, which suggests that these criteria cannot be maintained across all body sizes, instead maximum potential muscle power may vary with body size, producing an $n$-shaped relationship between power and body mass (M).

From our scaling model, we propose two alternative hypotheses. $\mathrm{H}_{1}$ : muscle power limits speed. Given this limitation, we would predict an $n$-shaped pattern with speed and body mass for swimming frogs, similar to that reported for terrestrial animals. $\mathrm{H}_{2}$ : factors other than muscle power limit speed. For example, if size-related bone stress is responsible for the $n$-shaped curve in terrestrial species, we would expect this effect to be removed for aquatic species. Thus, geometric scaling of muscle forces would predict a linear relationship between speed and body size for aquatic frogs.

Here we measure the performance of swimming $X$. laevis, with body mass varying from 1 to $184 \mathrm{~g}$ and, further, we test how morphological and physiological parameters scale with body size. We show that muscle-environment interactions limit the ability of muscle to operate at $1 / 3 V_{\max }$, predicting an 'optimal' body size for producing muscle power. Consequently, we show evidence for an $n$-shaped curve for swimming speed versus body mass.

\section{Results}

Swimming speed. On the basis of our geometric scaling argument, we expected that power and, therefore, swimming speed, would follow an $n$-shaped trend rather than increase linearly. Supporting our hypothesis, the fastest swimming speed for $X$. laevis was $2.43 \mathrm{~m} \mathrm{~s}^{-1}$ observed for an intermediately sized frog of $61 \mathrm{~g}$. The relationship between speed and mass was not linear, but was significantly better described by a second-order polynomial $\quad\left(\mathrm{AIC}_{\mathrm{lin}}=-30.98, \quad R^{2}=0.46 ; \quad \mathrm{AIC}_{\text {non-lin }}=-37.31\right.$, $\left.R^{2}=0.63\right)$. This indicated an optimal mass of $47.35 \mathrm{~g}$ at a speed of $1.52 \mathrm{~m} \mathrm{~s}^{-1}$ (Fig. 1b).

Geometric scaling of morphology. To further test muscle power limitations to swimming speed, we investigated the scaling of both physiological and morphological parameters. Morphological lengths of the PL muscle, tarsus and foot scaled as expected for a geometrically similar animal (Table 1), supporting assumptions in the model. Similarly, consistent with previous studies ${ }^{14}$, in vitro muscle experiments show the scaling of $V_{\text {max }_{\mathrm{rel}}}$, was not significantly different from $\mathrm{M}^{0}$ (Table 1 ). The mean $V_{\text {max }_{\text {rel }}}$ for $X$. laevis was $6.8 \pm 0.33 L_{\mathrm{m}} \mathrm{s}^{-1}$ ( \pm s.e., $n=10$; Fig. $2 \mathrm{a}$ ).

Counter to expectations, muscle force was $\propto M^{0.944}$ (Fig. 2b), significantly higher than $\mathrm{M}^{0.666}$ predicted for a geometrically similar animal (Table 1). Previously, the cross-sectional area of the PL has been shown to scale higher than expected $\left(\sim \mathrm{M}^{0.9}\right)$ due to a relatively low scaling exponent of muscle fibre length ${ }^{15}$.

Given the positive allometry of muscle force, we modified our scaling argument to determine how muscle-shortening velocity (therefore power) must scale with mass. To balance the forces on the foot, drag force must also scale more steeply than predicted $\left(\propto \mathrm{M}^{0.944}\right)$ to balance muscle force. Substituting $F_{\mathrm{drag}} \propto \mathrm{M}^{0.944}$ in equation (4) and using geometric scaling of out-lever (R) and foot area $\left(A_{\text {foot }}\right)$ yields a predicted scaling of ankle rotational velocity $(d \theta / d t) \propto M^{-0.194}$. This agrees closely with the scaling of empirical data for mean ankle rotational velocity $\left(\mathrm{M}^{-0.191}\right)$ and maximal ankle rotational velocity $\left(\mathrm{M}^{-0.205}\right)$ recorded in freely swimming frogs (Table 1 and Fig. 1c). Thus, both freely swimming frogs and in vivo data support our prediction that larger frogs rotate their ankles more slowly due to the scaling of fluid drag.

Using scaling data for ankle rotational velocity, we determined the scaling of muscle-shortening velocity via measurements of inlever and out-lever. Tendon travel experiments confirm that similar to muscle length and muscle out-lever, the scaling of the PL muscle in-lever was not significantly different from the predictions for a geometrically similar animal (Table 1 and Fig. 2a). Substituting ankle rotational velocity $\propto \mathrm{M}^{-0.191}$ and $r$ $\propto \mathrm{M}^{0.333}$ into equation (3), $V_{\text {abs }}$ should scale as $\mathrm{M}^{0.142}$, which is less steep than originally predicted $\left(\propto \mathrm{M}^{0.33}\right)$. As $V_{\text {max }_{\text {rel }}}$ was found to be independent of body size, and muscle length scaled geometrically, $V_{\text {max }_{a b s}}$ should scale as $\mathrm{M}^{0.333}$ (equation (2)). Consequently, $V / V_{\max }$ is expected to scale as $\mathrm{M}^{0.142} / \mathrm{M}^{0.333}$ $\propto \mathrm{M}^{-0.191}$, meaning that as frogs grow larger, muscles shift leftward on their power-velocity curve.

Scaling of muscle power. As a consequence of decreasing $V / V_{\max }$ with body size, muscle power should trace the predicted $n$-shaped power curve ${ }^{13}$. Figure $2 c$ shows the proportion of maximum power available to a muscle over the range of muscle-shortening velocities we have observed for frogs of different body masses (see 'In vivo muscle-shortening velocities' below). When these power values for frogs are compared with body mass (Fig. 2d), the curve of power versus mass corresponds well to the curve for swimming speed, predicting an optimum mass with respect to power of $47.27 \mathrm{~g}$, almost identical to the optimal mass predicted from swimming speeds ( $47.35 \mathrm{~g}$; Fig. 1b). To further compare the power-mass and speed-mass curves, we used the optimum value to separate small and large frogs. From this size-separated data, 

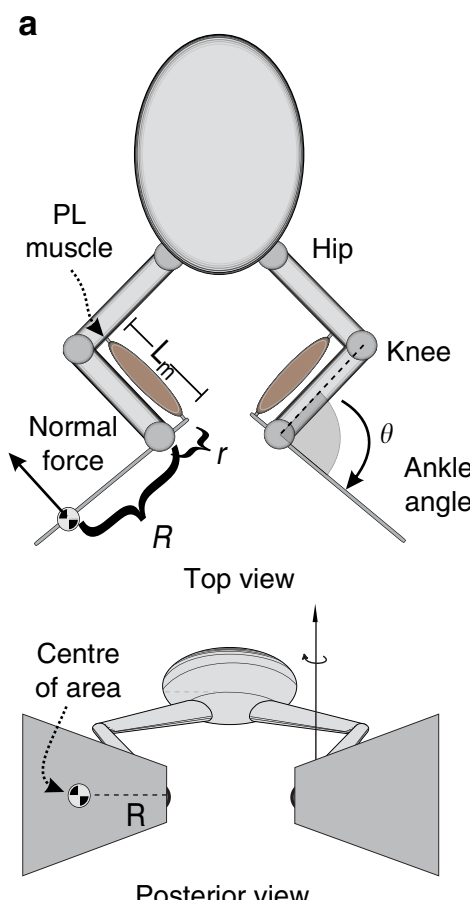
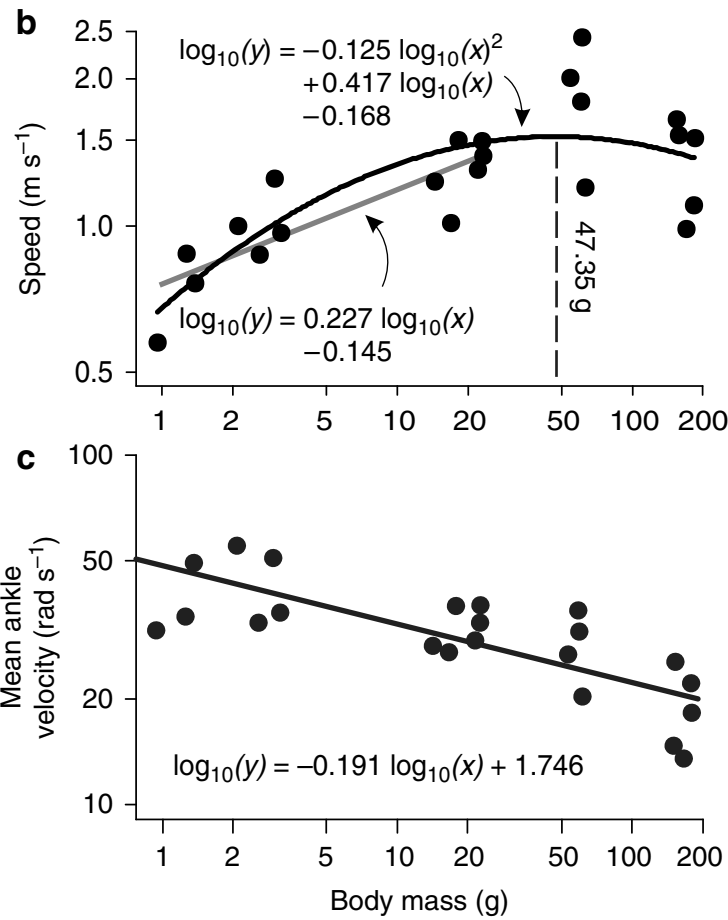

Figure 1 | Xenopus model and performance. (a) Simplified $X$. laevis model showing the attachment of the PL muscle, the muscle in-lever ( $r$ ), out-lever ( $R$ ), muscle length $\left(L_{m}\right)$ and ankle angle $(\theta)$. (b) Maximum swimming speed from 22 individual $X$. laevis versus mass showing optimum mass for speed (dashed line) from curvi-linear regression for all frogs (black line) and linear regression for small frogs ( $<47.35 \mathrm{~g}$; grey line). (c) Mean rotational velocity of the ankle during the fastest swimming trial for each individual versus mass.

\section{Table 1 | RMA scaling exponents against mass for Xenopus laevis.}

\begin{tabular}{|c|c|c|c|c|c|c|c|c|}
\hline Parameter $(y)$ & $n$ & $\boldsymbol{a}$ & $\boldsymbol{b}$ & $\boldsymbol{R}^{2}$ & P-value & Ho & $\boldsymbol{F}_{2, \mathbf{n}}$ & P-value \\
\hline Mean ankle velocity $\left(\mathrm{rads}^{-1}\right)$ & 22 & 1.746 & -0.191 & 0.562 & $<0.001$ & 0.333 & 15.175 & 0.001 \\
\hline Maximum ankle velocity $\left(\operatorname{rad~s}^{-1}\right)$ & 22 & 1.891 & -0.205 & 0.665 & $<0.001$ & 0.333 & 14.624 & 0.001 \\
\hline Plantaris longus muscle length (m) & 10 & -2.170 & 0.337 & 0.965 & $<0.001$ & 0.333 & 0.093 & 0.768 \\
\hline Tarsus length (m) & 20 & -2.287 & 0.322 & 0.982 & $<0.001$ & 0.333 & 1.152 & 0.297 \\
\hline Foot length (m) & 20 & -2.021 & 0.316 & 0.973 & $<0.001$ & 0.333 & 1.866 & 0.189 \\
\hline Foot area $\left(\mathrm{m}^{2}\right)$ & 19 & -4.258 & 0.685 & 0.995 & $<0.001$ & 0.666 & 2.377 & 0.142 \\
\hline Plantaris longus muscle in-lever (m) & 15 & -3.371 & 0.384 & 0.760 & $<0.001$ & 0.333 & 1.282 & 0.278 \\
\hline Isometric muscle force $(N)$ & 8 & -0.551 & 0.944 & 0.991 & $<0.001$ & 0.666 & 87.469 & $<0.001$ \\
\hline$V_{\text {max }_{\text {rel }}}\left(L_{m} s^{-1}\right)$ & 10 & 0.654 & 0.1355 & 0.032 & 0.620 & 0 & - & - \\
\hline
\end{tabular}

linear regression predicted a positive relationship for the speed of small frogs $\left(R^{2}=0.72, P<0.001\right.$; analysis of variance), increasing as $\mathrm{M}^{0.227}$ (Fig. 1b). The regression describing larger frogs was not significant ( $R^{2}=0.35, P=0.095$; analysis of variance). The scaling of power with mass for small frogs was $M^{0.201}$ (Fig. 2d), not significantly different to that of swimming speed with mass $\left(\mathrm{F}_{2,26}=0.490, P=0.474\right.$; Log-likelihood). Thus, the change in swimming speed with mass can be completely explained by the variation in muscle power with size.

\section{Discussion}

We have shown that the scaling of muscle-shortening velocity differentially limits the capacity for producing power in vitro. As muscle power is maximal at $\sim 1 / 3 V_{\max }$, the scaling of muscle force coupled with fluid drag force implies that this optimal shortening velocity for power cannot be reached for all body sizes, but instead follows an ' $n$-shaped' pattern. This pattern is shown not only in our in vitro muscle results, but is mirrored in whole animal swimming speed, specifically the rate of increase of swimming speed with mass, the mass at peak swimming speed and also the subsequent decline of swimming speed with further mass increases. The correspondence between our predicted scaling for muscle power and our observed scaling for swimming speed suggests that the $n$-shaped power-velocity curve limits swimming speed.

The $n$-shaped speed-mass curve predicted by our current model is in contrast to previous predictions of locomotor speed versus body size. Earlier studies suggested that as muscle force scales with cross-sectional area of the muscle, and stride frequency scales with the inverse of such, maximum speed was thought to be independent of body mass ${ }^{16}$. Since then, several other variations of this scaling model have predicted a positive linear relationship between speed and size $^{1}$. In contrast with prior predictions, we have shown that at least for speed in aquatic systems, it is the interaction between a muscles power-velocity 
a
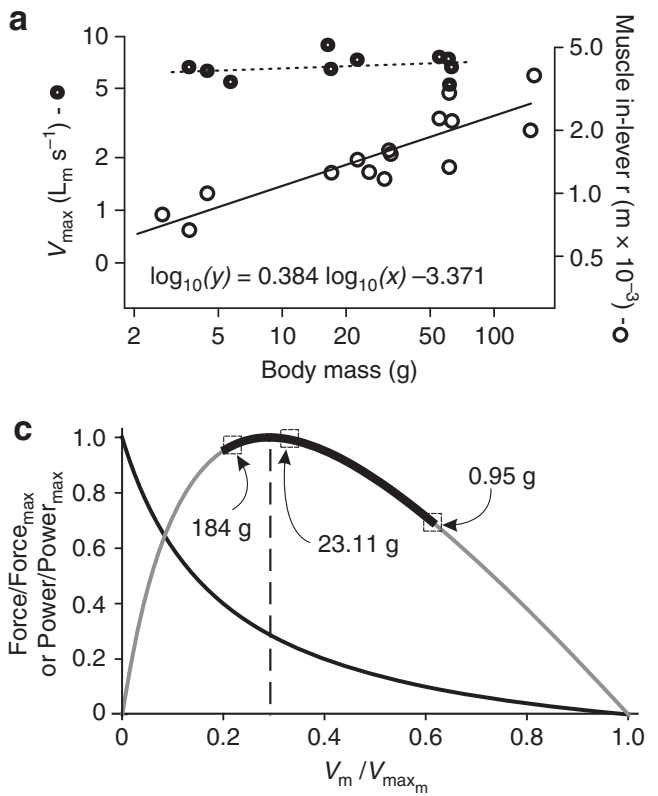

b
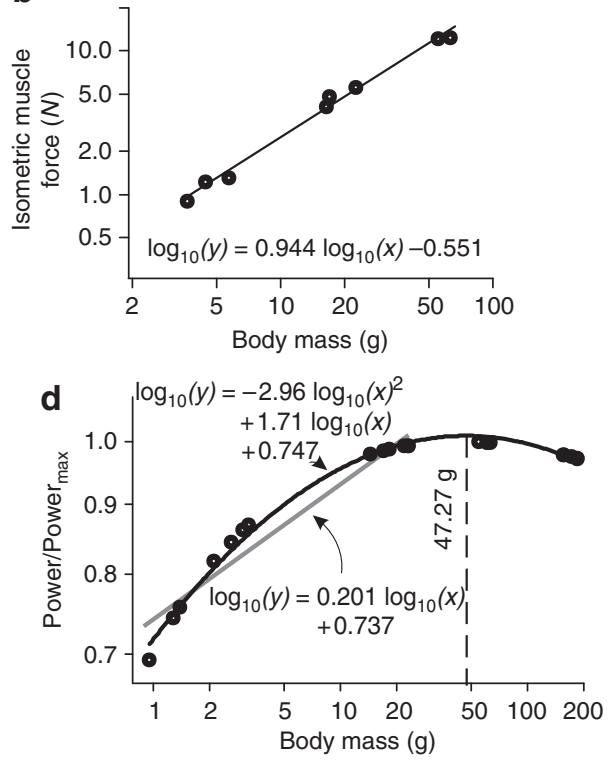

Figure 2 | In vitro results for X. laevis PL muscle. (a) Maximum muscle-shortening velocity (closed circles) and in-lever (open circles) versus body mass. Dashed line indicates slope not significantly different from 0. (b) Isometric muscle force versus body mass (c) Force-velocity (thin black) and power-velocity (thin grey) curves for the PL muscle. Dashed line indicates optimal power at $\sim 0.3 V_{\max }$. The thick black line indicates the range of maximum muscle power available for frogs based on the scaling of mean muscle velocity (Fig. 1c), arrows indicate three example body masses. (d) Maximum muscle power available from power-velocity curve (Fig. 2c) versus body mass, estimated from mean muscle velocity. Optimal mass for muscle power indicated by dashed line. Linear regression for small frogs $(<47.27 \mathrm{~g})$ indicated by grey line.

curve with stride frequency, which may ultimately limit speed and result in the observed $n$-shaped pattern.

This muscle power limitation to swimming speed has important implications for the evolution of speed in aquatic systems. As swimming speed is often considered an ecologically relevant task for catching prey and avoiding predators, then larger body sizes may be selected against in these systems. If this scaling relationship is ecologically relevant, we may then expect to see the size distribution in natural populations centred on the optimal size for speed. Studies in wild population of Xenopus seem to support this hypothesis. Mean body size for three different reference sites in South Africa was $39 \mathrm{~g}$ for female frogs ${ }^{17}$, close to the predicted mass of $42 \mathrm{~g}$, and certainly less than the maximum body size possible.

The simplicity of this model suggests that these principles may be applicable to a range of animals. Most specifically, this model may be relevant for swimmers, where propulsion is 'rowing-like' by simple drag-based rotation, for example, in turtles ${ }^{18}$, aquatic insects ${ }^{19}$ and rowing fish ${ }^{20}$. The model may also be extended to other species of swimming frogs ${ }^{21}$, although some caution should be used in that the current model only considers rotational drag. Although this is appropriate for X. laevis for which nearly $100 \%$ of the thrust is derived from rotational foot motion, in other frog species between $15-25 \%$ of the thrust may be derived from translational foot motion, suggesting multiple muscles contribute to thrust. The outcome of variation in neuro-muscular coordination of multiple muscles is unknown but is likely to be complex. For example, if the extent to which frogs generate propulsion by foot translation versus rotation shifts over body size, the scaling of swimming speed might differ from our simple model. Such effects of the scaling of limb kinematics patterns should be investigated in future work.

Of additional interest beyond swimming mechanics is the degree to which this finding can be related back to terrestrial studies, which also show the $n$-shaped trend between maximum speed and mass. Currently, the best explanation for this trend in terrestrial systems suggests speed may increase with body size until a certain safety factor is reached, above which speed must decrease to reduce the peak forces acting on limb bones $5,6,22$. However, with increasing body size, limb beat frequency has been shown to decrease for both mammals ${ }^{23}$ and lizards ${ }^{24}$, reflecting the changes in ankle rotation seen in the current study. Assuming that limb kinematics, posture and motor coordination patterns do not change dramatically over size, we would expect muscle power to similarly rise and fall as the operating point on the powervelocity curve shifts. This change in muscle power over size may then contribute to the rise and fall of speed with size in these terrestrial animals. Thus, we propose that future studies may apply our model more broadly to investigate the muscular limits of running speed.

In contrast with our scaling regime, several studies on terrestrial lizards reported that power did not appear to limit speed. Power output was shown to increase as lizards ran up steeper inclines, and for animals loaded with weights ${ }^{6,25}$; therefore, it was suggested that lizards running on level surfaces were doing so at submaximal power ${ }^{7,8}$. However, within these trials, stride frequency also varied significantly with the loading regime ${ }^{7}$. Along with changes in external loading, the change in stride frequency seen within these studies may have shifted the muscle along its power-velocity curve to a shortening velocity more favourable for power production, explaining changes in power output. As the current study has illustrated, performance may not be limited by maximum power output possible per se, but rather the maximum power output obtainable at a given stride frequency ${ }^{26}$.

We have proposed a novel scaling principle predicting that relative muscle-shortening velocity $\left(V / V_{\max }\right)$ decreases with body mass. For frogs, the implications of such scaling are dramatic; the negative scaling of $V / V_{\max }$ limits power, and therefore the swimming speed. The simplicity of our model suggests that 
geometrically scaled drag-based swimmers, in general, are also subject to such scaling principles. For future studies, we propose that our scaling principle may more broadly apply to running animals, despite mechanical differences between land and water. Regardless of locomotor mode, muscle power may still impose a limit to speed in each case, although the constraint does not lie in the maximal ability of the muscle to produce power, but rather the ability of the muscle to produce power as the muscularskeletal system interacts with the external environment.

\section{Methods}

Scaling model. Using geometric scaling principles, we show why muscles cannot produce maximum power at all body sizes. For our model, we use scaling in proportion to body mass as $\propto \mathrm{M}^{b}$ where $b$ is the slope on a log-log plot. On the basis of geometry, we apply three scaling rules: mass (for example, muscle mass) $\alpha \mathrm{M}^{1}$, length (for example, limb length) $\alpha \mathrm{M}^{1 / 3}$ and area (for example, webbed foot area) $\alpha \mathrm{M}^{2 / 3}$

Simple models have often served to reveal general principals in complex systems ${ }^{27-29}$. We follow these examples and have modelled the movement of $X$. laevis as a simple muscle lever system, powered by the plantaris muscle, with a rigid flat fin rotating around about the ankle joint (Fig. 1a). To determine the consequences of geometric scaling on muscle function, we express ankle rotational velocity in terms of muscle-shortening velocity. Next, we determine how ankle velocity (and therefore muscle velocity) relates to propulsive drag force by rotating the feet.

Our scaling argument is as follows. First, we assume that swimming animals of all sizes maximize power by maintaining $V / V_{\max }=0.3$. Second, we describe the scaling of foot kinematics required to satisfy the above assumption. Third, based on the scaling of fluid drag, we refute the above assumption. Fourth, we propose a more plausible scaling principle that predicts allometric scaling of $V / V_{\max }$, rather than geometric scaling.

If muscles do produce maximum muscle power over all body sizes, we would expect all muscles to operate at a shortening velocity of $1 / 3 V_{\max } 13$, that is,

$$
V_{\text {rel }} / V_{\max _{\mathrm{rel}}}=V_{\mathrm{abs}} / V_{\max _{\mathrm{abs}}} \alpha \cdot \mathrm{M}^{0}
$$

where $V_{\text {rel }}$ and $V_{\text {abs }}$ represent the relative (muscle lengths per second, $L_{\mathrm{m}} \mathrm{s}^{-1}$ ) and absolute $\left(\mathrm{m} \mathrm{s}^{-1}\right)$ muscle-shortening velocity, respectively. For a large range of muscle types, the relative maximum shortening velocity of vertebrate muscle $\left(V_{\max _{\mathrm{rel}}}\right)$ is nearly independent of body size $\mathrm{e}^{14}$. If this is true for frogs, then the absolute maximum value of muscle-shortening velocity $\left(V_{\max _{a b s}}\right)$ must be proportional to $\mathrm{M}^{1 / 3}$, as we can expect absolute maximum shortening velocity to be a product of relative maximum shortening velocity and muscle length,

$$
V_{\text {max }_{\mathrm{abs}}}=V_{\text {max }_{\mathrm{rel}}} \cdot L_{\mathrm{m}}
$$

where $V_{\text {max }_{\text {rel }}}$ scales as $\sim \mathrm{M}^{0}$, whereas muscle length $\left(L_{\mathrm{m}}\right)$ is proportional to $\mathrm{M}^{1 / 3}$ for geometrically symmetrical animals ${ }^{30}$, leaving equation (2) $\alpha \mathrm{M}^{0} \mathrm{M}^{1 / 3}=\mathrm{M}^{1 / 3}$. By substituting $V_{\text {max }_{a b s}} \alpha \mathrm{M}^{1 / 3}$ into equation (1), we expect absolute muscle-shortening velocity $\left(V_{\text {abs }}\right.$ ) to also scale positively as $V_{\text {abs }} \alpha \mathrm{M}^{1 / 3}$ to maintain $V / V_{\max } \alpha \mathrm{M}^{0}$. Using this prediction for muscle-shortening velocity $\left(V_{\mathrm{abs}}\right)$, the scaling of ankle rotational velocity is given as:

$$
\text { Ankle rotational velocity }(d \theta / d t) \approx V_{\mathrm{abs}} / r
$$

where $r$ is the muscle in-lever, $\theta$ is the ankle angle and $t$ is time in seconds (Fig. 1a). As we expect muscle in-lever to scale with length as $\mathrm{M}^{1 / 3}$, we can substitute $r$ and $V_{\text {abs }}$ into equation (1.3) to obtain ankle velocity $\alpha \mathrm{M}^{1 / 3} / \mathrm{M}^{1 / 3}=\mathrm{M}^{0}$. Hence, to maintain $V / V_{\max }=0.3$ for optimal muscle power, ankle rotational velocity should be independent of body size. However, this conclusion is challenged when we consider the drag force required to move a foot through water. At high Reynolds numbers, propulsive drag $\left(F_{\text {drag }}\right)$ is proportional to the square of velocity ${ }^{31}$ :

$$
F_{\mathrm{drag}}=\frac{1}{2} \cdot p \cdot C_{\mathrm{d}} \cdot A_{\mathrm{foot}} \cdot(R \cdot d \theta / d t)^{2}
$$

where $p$ is the water density, $C_{\mathrm{d}}$ is the foot-drag coefficient, $A_{\text {foot }}$ is the foot area, $R$ is the distance from the ankle to the fluid centre of pressure and velocity is the ankle rotational velocity. Substituting geometric scaling equations for length and area, $C_{\mathrm{d}} \alpha \mathrm{M}^{0}$, and $d \theta / d t$ as $\alpha \mathrm{M}^{0}$ predicted above, then drag force on the foot is expected to scale with positive allometry: $F_{\mathrm{drag}} \alpha \mathrm{M}^{0} \cdot \mathrm{M}^{2 / 3} \cdot\left(\mathrm{M}^{1 / 3} \cdot \mathrm{M}^{0}\right)^{2} \alpha \mathrm{M}^{4 / 3}$

As muscle force $\left(F_{\text {muscle }}\right)$ must resist this drag force, our assumption that all muscles operate at the peak of their power-velocity curve has created a force balance problem. Muscle force typically scales with muscle cross-sectional area ${ }^{16}$ $\left(\alpha \mathrm{M}^{2 / 3}\right)$, requiring that $F_{\text {drag }}$ also scale as $\mathrm{M}^{2 / 3}$ rather than $\mathrm{M}^{4 / 3}$, to balance the forces. Therefore, $R, A_{\text {foot }}$ or ankle rotational velocity must scale differently to our above expectations. Given that scaling of frog hindlimbs has been shown previously ${ }^{30}$ supporting geometric scaling for $R\left(\alpha \mathrm{M}^{1 / 3}\right)$ and $A_{\text {foot }}\left(\alpha \mathrm{M}^{2 / 3}\right)$, we predict ankle rotational velocity must scale with negative allometry (that is, decrease with mass) to balance the forces. Specifically, we predict ankle rotational velocity $(d \theta / d t)$ must scale as $\mathrm{M}^{-1 / 3}$, enabling foot drag $\alpha \mathrm{M}^{2 / 3}$
The implication of this negative scaling of ankle rotational velocity is profound. As the body size increases, $V / V_{\max }$ cannot be maintained at $1 / 3 V_{\max }$ where power is maximal. To summarize our scaling argument, we work backwards from equation (3). The negative allometry of ankle rotational velocity $\left(d \theta / d t \alpha \mathrm{M}^{-1 / 3}\right)$ predicts $V_{\mathrm{abs}} \alpha \mathrm{M}^{0}$. Consequently, $V_{\max _{\mathrm{abs}}} \alpha \mathrm{M}^{1 / 3}$ (equation (2)), predicting that $V_{\mathrm{abs}} /$ $V_{\max _{a b s}} \alpha \mathrm{M}^{-1 / 3}$. This negative scaling of $V / V_{\max }$ suggests that the muscles of different-sized frogs operate at different regions of their power-velocity curve, which will limit the ability of muscle to realize its maximum potential power. Consequently, we expect an $n$-shaped relationship between body size and maximum possible power, initially increasing until body size coincides with $1 / 3$ $V_{\max }$, then decreasing as mass further increases.

Animal procurement and maintenance. X. laevis Daudin $1802(n=22$, body mass range 0.95-184 g) were obtained from Xenopus Express Inc. (Plant City, FL, USA). Animals were housed in aquaria and maintained at $20-22^{\circ} \mathrm{C}$ under a $12: 12 \mathrm{~h}$ light:dark cycle, and fed twice per week. Each animal was weighed before experiments $( \pm 0.01 \mathrm{~g})$ and the length of the tarsus and the foot (metatarsals + phalanges) were measured $( \pm 0.01 \mathrm{~mm})$. All procedures on animals were approved by the Institutional Animal Care and Use Committee at Harvard.

Swimming speed and kinematics. Maximum swimming speed and kinematics for frogs $<60 \mathrm{~g}$ were measured in a Plexiglas racetrack $(15 \times 180 \mathrm{~cm})$ and frogs $>60 \mathrm{~g}$ were measured in a larger tank $(90 \times 180 \mathrm{~cm})$ at $21-22^{\circ} \mathrm{C}$. Shallow water (depth $10-12 \mathrm{~cm}$ ) encouraged horizontal swimming. Frogs were swum down the track five to six times and filmed using a Photron Fastcam SA-3 (Photron USA Inc., CA, USA) at $250 \mathrm{fps}$. The snout tip was digitized for each trial using the MATLAB script DLTdv3 (version 3$)^{32}$ and smoothed using the spaps.m smoothing spline in MATLAB. Maximum swimming speed was calculated from the instantaneous difference between smoothed position data. Akaike's information criterion (AIC, implemented in R (Ver 2.13.0), was used to determine whether linear or nonlinear (second-order polynomial) regressions described the relationship with speed.

Measuring maximum performance across a large body size range can be difficult, as variation in body size may be correlated with variation in behavioural strategies. We therefore undertook several precautionary evaluations to ensure we were measuring maximum speeds. We have followed the advice of Losos et al. ${ }^{33}$ who suggest that two common flaws can be seen in many published papers: data are included from individuals obviously performing submaximally and not enough trials are conducted to provide an accurate estimate of maximal capabilities.

To overcome these problems, we excluded any trials where frogs failed to swim in a straight line, or hit the edges of the swim track, to remove submaximal trials. We further measured a large number of trials. We used 251 trials for 22 individual frogs (mean trials per individual $=11.4$ ), choosing the maximum speed for each individual (Fig. 3a).

We further tested the variation in submaximal trials for each body size. If swimming speed was affected by variation in behaviour, with the largest frogs showing greater variation in behaviour, we might expect that the s.d. in speeds would be highest for large frogs. This does not appear to be the case (Fig. 3b). The s.d. does not change consistently with body size, nor do the largest frogs show the highest variation in swim speeds, in many cases the deviation being lower than that for the smallest frogs. This suggests that large frogs are swimming close to some physical limits of their ability rather than behavioural effects.

For the fastest trial for each individual, mean ankle rotational velocity (averaged over the time duration of the power stroke) was calculated from video sequences by digitizing the ankle joint, tibia and the tip of the longest toe.

In vitro muscle preparation and force-velocity measurement. As $X$. laevis swim by ankle rotation ${ }^{9}$, we focused on the PL muscle. We measured $V_{\max }$ and isometric muscle force $\left(F_{\mathrm{o}}\right)$ for a subset $(n=10)$ of the frogs used in the swimming trials. Frogs were double pithed with a 21-gauge syringe needle, and the PL muscle length was recorded by positioning the hip, knee, ankle and metatarsal joints at $90^{\circ}$ and measuring the longest proportion of the muscle between the tendon and the aponeurosis. The PL was then removed, keeping the proximal attachment at the knee intact, by cutting the femur $\sim 0.5 \mathrm{~cm}$ from the knee joint. Surgical suture (Vicryl 4-0, braided, Ethicon) was threaded though the femur to anchor the proximal end of the muscle. At the distal end of the PL muscle, suture was threaded through and around the PL muscle just proximal to the PL tendon. The muscle was then mounted to the ergometer, with the proximal end tied to a stiff metal pin embedded in Plexiglass, and the distal suture tied to the small hole in the lever on the servo motor (305C-LR, Aurora Scientific Inc., Aurora, ON, Canada). This setup was then bathed in oxygenated amphibian ringers solution (Carolina Biological, Burlington, NC, USA) at $22^{\circ} \mathrm{C}$.

Starting tension for isotonic experimental contractions was determined experimentally using isometric twitch contractions. The passive force at which twitch muscle forces were maximal was used for starting tension. During experiments, muscle was maximally stimulated using stainless steel plate electrodes with a 150 -ms pulse train at supramaximal voltage $(18 \mathrm{~V})$ at a spike frequency of $250 \mathrm{~Hz}$ based on in vivo electromyography patterns previously observed in the PL muscle of $X$. laevis ${ }^{10}$. Stimulation pulses of 1 -ms width from an A/D board were 

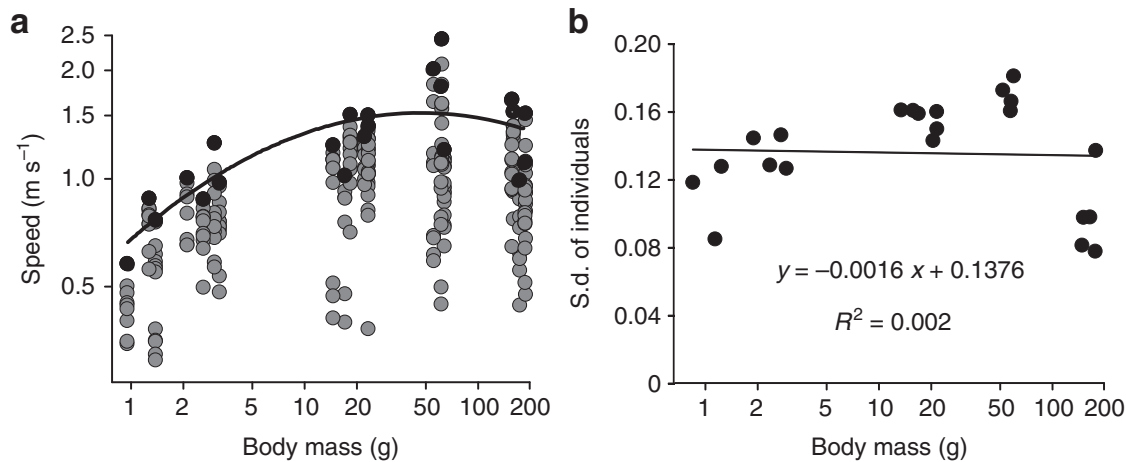

Figure 3 | Estimating maximum speed during swimming trials. (a) Swimming speed with mass for all trials during experimentation. Solid black circles represent maximum swim speeds for each individual, whereas grey shaded circles are submaximal swim speeds; (b) standard deviation for swimming speed with mass. Line represents the least-squares regression.

a

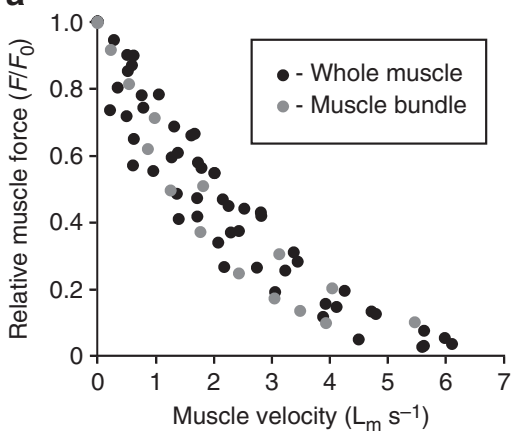

b

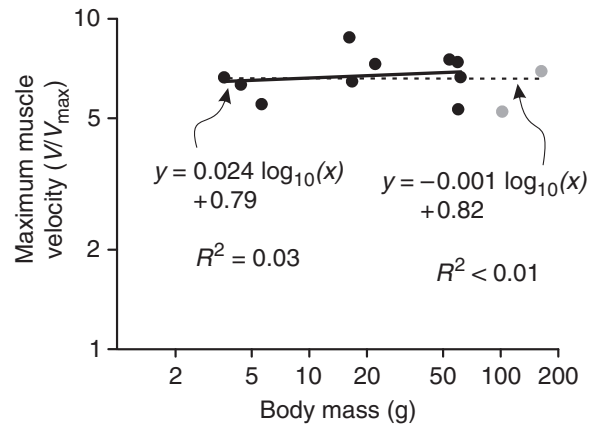

Figure 4 | Estimating muscle degradation with size for in vitro muscle experiments. (a) Curvi-linear relationship between relative muscle force and muscle velocity for whole-muscle preparations (solid black circles) and dissected muscle bundles (grey circles). (b) Relationship between maximum muscle velocity and mass for whole-muscle preparations (solid black circles) and dissected muscle bundles (grey circles). Solid black line is regression for whole-muscle preps, dashed line shows regression for both whole-muscle preps and muscle bundle preps combined. Results and $R^{2}$ shown for the solid line (left) and the dashed line (right).

amplified by an OPA549T op-amp (Texas Instruments, Dallas, TX, USA) powered by a Sorensen LS 18-5 power supply (AMETEK Programmable Power, Inc., CA, USA). The muscle was allowed to rest for $10-15$ min between contractions. The isotonic force was varied in $0.1 \mathrm{~N}$ intervals, until the muscle was unable to move the force lever (that is, an isometric contraction), and both muscle force and muscle length were recorded with a NI-6289 data acquisition system (National Instruments, Austin, TX, USA). To calculate $V_{\text {max }_{\mathrm{abs}}}$, the velocity of shortening $\left(V_{\text {abs }}\right)$ at each isotonic force $\left(F_{\text {muscle }}\right)$ was fitted using the equation (5),

$$
F_{\text {muscle }}=\left(b \cdot F_{\mathrm{o}}-a \cdot V_{\mathrm{abs}}\right) /\left(V_{\mathrm{abs}}+\mathrm{b}\right)
$$

where $a$ and $b$ are constants specific to the muscle and $F_{\mathrm{o}}$ is the maximum isometric muscle force ${ }^{19,31} . V_{\text {max }_{a b s}}$ is then calculated where the force drops to zero $\left(V_{\text {max }_{a b s}}=b \cdot F_{\mathrm{o}} / a\right)$.

Muscle bundle experiments. To verify that our whole-muscle tissue preparations did not degrade due to the large cross-sectional area of the muscle, we performed identical experiments on muscle bundles. In the largest frogs (total body mass 105 and $168 \mathrm{~g}$ ), we isolated muscle bundles $\left(0.432-0.695 \mathrm{~g}\right.$ in mass; $\sim 0.1-0.2 \mathrm{~cm}^{2}$ cross-sectional area). Under a dissecting microscope, the broad plantaris muscle was cut using a scalpel blade such that a section containing the longest fibres remained. All remaining damaged fibres were carefully removed with $2 \mathrm{~mm}$ spring scissors. To determine that the tissue did not degrade, we verified that the maximum tetanic isometric force remained within $10 \%$ of its initial value throughout the experiment. In addition, we verified that muscle bundles produced similar peak isometric stress $\left(\sim 25-30 \mathrm{~N} \mathrm{~cm}^{-2}\right)$ as found in whole-muscle preparations. Finally, we compared our muscle bundle experiments to the whole-muscle results. This showed good agreement between whole-muscle experiments and muscle bundle experiments for force velocity (Fig. 4a) and $V_{\text {max }_{\text {rel }}}$ (Fig. 4b).

Elastic energy storage. To determine the extent that elastic energy storage has in this system, we re-examined data from Richards and Beiwener ${ }^{10}$ who simultaneously measured both muscle length (using sonomicrometry crystals) and ankle angle kinematics in $X$. laevis. If elastic energy storage is present in the plantaris muscle, we would expect the relationship to be 'L-shaped', with a period showing a change in muscle length but no corresponding change in ankle angle (indicating the tendon is lengthening), and a period showing a change in ankle angle without a corresponding change in muscle length (indicating tendon recoil). This would be similar to the results shown by Astley and Roberts ${ }^{34}$ for the vertebrate catapult in bullfrogs (see Fig. 2 in Astley and Roberts ${ }^{34}$ ). Alternatively, if energy storage has little or no role in Xenopus swimming, we would expect a linear relationship between muscle length and ankle angle, indicating a change in muscle length corresponds directly to a change in ankle angle. Figure 5 shows the results from the reanalysis from Richards and Beiwenier ${ }^{10}$, which appear to support a linear relationship between ankle angle and muscle length, suggesting little influence of elastic energy storage.

In addition, evidence from mathematical modelling of a swimming limb powered by a muscle-tendon system suggests negligible effects of elastic recoil under natural conditions. Specifically, muscle-tendon simulation conditions based on $X$. laevis plantaris properties predict that the mechanical power contribution from the recoiling tendon does not substantially add to the power exerted by the muscle $^{35}$.

Tendon travel methods. The tendon travel method was used to determine the muscle moment arm for the PL muscle. The muscle moment arm was calculated from the slope of the relationship between the length change in the PL muscle/ tendon unit as the ankle joint is rotated ${ }^{13,34}$. To measure these movements, leg bones were dissected out, keeping the joint tissue at the ankle intact. The tibiofibula was firmly attached to a surface, using suture thread, such that the flexion/ extension axis was horizontal. Pins were forced into the tibio-fibula and tarsus segments to serve as markers for bone position. The proximal attachment of the PL muscle/tendon unit was severed and a long suture thread (Vicryl 4-0, braided, Ethicon) was attached to the distal end of the PL muscle. A 150-g mass was attached to the end of the suture to apply a constant load to the tendon. The foot was then flexed and extended while being filmed with a Photron fastcam (SA-3) at 

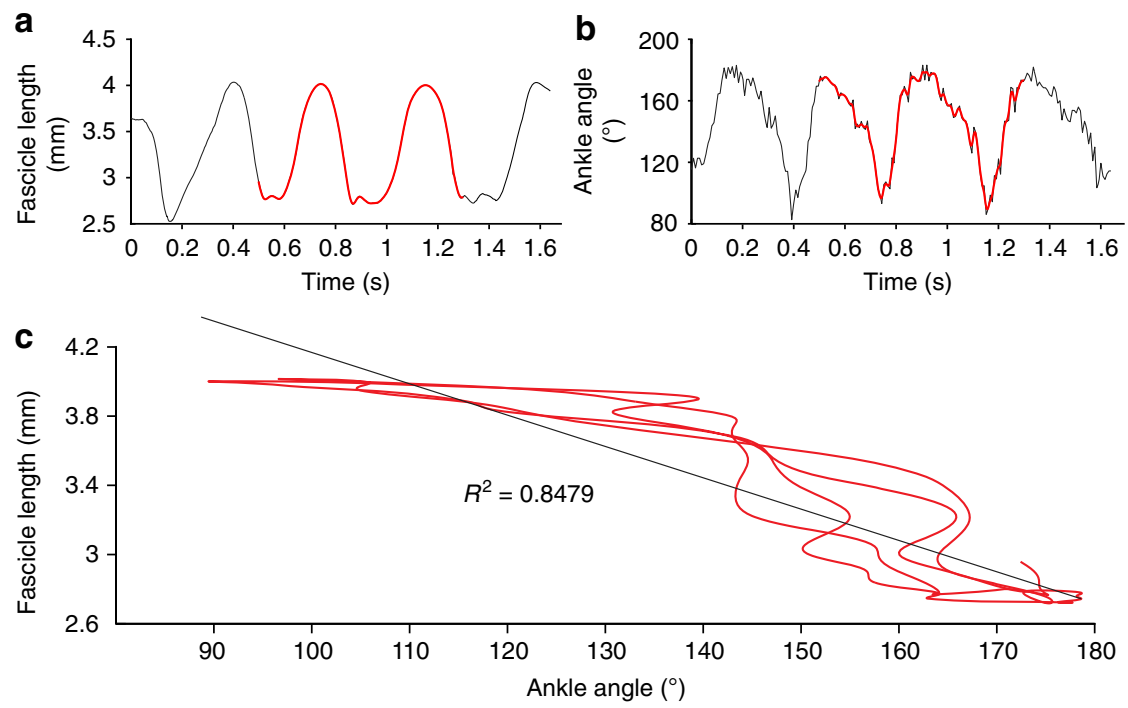

Figure 5 | Muscle fascicle length changes and ankle angle changes in freely swimming frogs. Data from Richard and Biewener ${ }^{9}$. (a) Time series of plantaris muscle length changes over several strokes. (b) Corresponding time series of ankle angle changes throughout the strokes. (c) Relationship between muscle length changes and ankle angle for the red highlighted sections from both $\mathbf{a}$ and $\mathbf{b}$.

$250 \mathrm{~Hz}$. Markers on the tarsus, tibio-fibula and the suture thread were then digitized to track displacement versus ankle angle.

In vivo muscle-shortening velocities. To determine the relative muscle velocity $\left(V_{\text {rel }}\right)$ for each individual, we used the mass of each individual entered into the scaling equations for mean angular velocity (Table 1$)$ and in-lever $\left(r ; \log _{10}\right.$ $r=0.333 \cdot \log _{10}$ mass-0.3), entered into equation (3). This was divided by muscle length as predicted from observed scaling (Table 1$) . V / V_{\max }$ for each individual was then calculated by dividing through by $V_{\max }$, which was constant with mass, as $6.8 \mathrm{~L}_{\mathrm{m}} \mathrm{s}^{-1}$ (see Results). Following this, muscle power for each individual could be calculated using the power-velocity curve based on Daniel ${ }^{31}$ as shown below.

$$
\text { Power } / \text { Power }_{\max }=\left[V_{\text {rel }} \cdot\left(V_{\text {max }_{\text {rel }}}-V_{\text {rel }}\right) /\left(V_{\text {max }_{\text {rel }}}+5 \cdot V \mathrm{~m}\right)\right] / \text { Power }_{\max }
$$

Statistics. All values were $\log _{10}$-transformed and scaling exponents for $V_{\text {max }}, F_{0}$, muscle length, in-lever, tarsus, foot length and area were determined using model II reduced major axis (RMA) regression. For each regression, the slope was tested against the expectation of isometric growth ${ }^{1}$. RMA slope estimates, confidence intervals and comparisons between the RMA slopes and expectations (using the likelihood ratio test) were implemented using the programme SMATR $^{36}$

\section{References}

1. McMahon, T. A. Muscles, Reflexes, and Locomotion (Princeton University Press, 1984)

2. Clemente, C. J., Thompson, G. G. \& Withers, P. C. Evolutionary relationships of sprint speed in Australian varanid lizards. J. Zool. 278, 270-280 (2009).

3. Garland, T. The relation between maximal running speed and body mass in terrestrial mammals. J. Zool. 199, 157-170 (1983).

4. Clemente, C. J., Withers, P. C. \& Thompson, G. G. Optimal body size with respect to maximal speed for the yellow-spotted monitor lizard (Varanus panoptes; Varanidae). Physiol. Biochem. Zool. 85, 265-273 (2012).

5. Clemente, C. J., Withers, P. C., Thompson, G. G. \& Lloyd, D. Evolution of limb bone loading and body size in varanid lizards. J. Exp. Biol. 214, 3013-3020 (2011).

6. Biewener, A. A. Scaling body support in mammals: limb posture and muscle mechanics. Science 245, 45 (1989).

7. Irschick, D. J., Vanhooydonck, B., Herrel, A. \& Andronescu, A. Effects of loading and size on maximum power output and gait characteristics in geckos. J. Exp. Biol. 206, 3923-3934 (2003).

8. Farley, C. T. Maximum speed and mechanical power output in lizards. J. Exp. Biol. 200, 2189-2195 (1997).

9. Richards, C. T. Kinematics and hydrodynamics analysis of swimming anurans reveals striking inter-specific differences in the mechanism for producing thrust. J. Exp. Biol. 213, 621-634 (2010).
10. Richards, C. T. \& Biewener, A. A. Modulation of in vivo muscle power output during swimming in the African clawed frog (Xenopus laevis). J. Exp. Biol. 210 3147-3159 (2007).

11. Gordon, A., Huxley, A. F. \& Julian, F. The variation in isometric tension with sarcomere length in vertebrate muscle fibres. J. Physiol. 184, 170-192 (1966).

12. Hill, A. The heat of shortening and the dynamic constants of muscle. Proc. $R$ Soc. Lond. Ser. B Biol. Sci. 126, 136-195 (1938).

13. Lutz, G. J. \& Rome, L. C. Built for jumping: the design of the frog muscular system. Science 263, 370-372 (1994).

14. Medler, S. Comparative trends in shortening velocity and force production in skeletal muscles. Am. J. Physiol. Regul. Integr. Compar. Physiol. 283, R368-R378 (2002).

15. Pollock, C. M. \& Shadwick, R. E. Allometry of muscle, tendon, and elastic energy storage capacity in mammals. Am. J. Physiol. Regul. Integr. Compar. Physiol. 266, R1022-R1031 (1994).

16. Hill, A. V. The dimensions of animals and their muscular dynamics. Sci. Prog. 38, 209-230 (1950).

17. Du Preez, L. H. et al. Population structure of the African Clawed Frog (Xenopus laevis) in maize-growing areas with atrazine application versus non-maizegrowing areas in South Africa. Afr. J. Herpetol. 54, 61-68 (2005).

18. Pace, C. M., Blob, R. W. \& Westneat, M. W. Comparative kinematics of the forelimb during swimming in red-eared slider (Trachemys scripta) and spiny softshell (Apalone spinifera) turtles. J. Exp. Biol. 204, 3261-3271 (2001).

19. Daniel, T. L. in Biological Fluid Dynamics Vol. 49 (eds Ellington, C. P. \& Pedley, T. J.) 61-89 (Company of Biologists, 1995).

20. Blake, R. W. The mechanics of labriform locomotion I. Labriform locomotion in the angelfish (Pterophyllum eimekei): an analysis of the power stroke. J. Exp. Biol. 82, 255-271 (1979).

21. Gal, J. M. \& Blake, R. W. Biomechanics of frog swimming: I. Estimation of the propulsive force generated by Hymenochirus boettgeri. J. Exp. Biol. 138, 399-411 (1988).

22. Farley, C. T. \& Taylor, C. R. A mechanical trigger for the trot-gallop transition in horses. Science 253, 306-308 (1991).

23. Heglund, N. C., Taylor, C. R. \& McMahon, T. A. Scaling stride frequency and gait to animal size: mice to horses. Science 186, 1112-1113 (1974).

24. White, T. D. \& Anderson, R. A. Locomotor patterns and costs as related to body size and form in teiid lizards. J. Zool. 233, 107-128 (1994).

25. Irschick, D. J., Bocchi, S. \& Full, R. J. Does power limit climbing performance? Loading small climbing lizards. Am. Zool. 41, 1481 (2001).

26. Medler, S. \& Hulme, K. Frequency-dependent power output and skeletal muscle design. Compar. Biochem. Physiol. A Mol. Integr. Physiol. 152, 407-417 (2009).

27. Aerts, P. \& Nauwelaerts, S. Environmentally induced mechanical feedback in locomotion: frog performance as a model. J. Theor. Biol. 261, 372-378 (2009).

28. Galantis, A. \& Woledge, R. C. The theoretical limits to the power output of a muscle-tendon complex with inertial and gravitational loads. Proc. R. Soc. Lond. Ser. B Biol. Sci. 270, 1493-1498 (2003).

29. Richards, C. T. \& Clemente, C. J. Built for rowing: frog muscle is tuned to limb morphology to power swimming. J. R. Soc. Interface 10, 20130236 (2013). 
30. Marsh, R. Jumping ability of anuran amphibians. Adv. Vet. Sci. Comp. Med. 38, 51-111 (1994).

31. Daniel, T. L. Unsteady aspects of aquatic locomotion. Am. Zool. 24, 121-134 (1984).

32. Hedrick, T. L. Software techniques for two-and three-dimensional kinematic measurements of biological and biomimetic systems. Bioinspir. Biomim. 3, 034001 (2008)

33. Losos, J. B., Creer, D. A. \& Schulte, J. A. Cautionary comments on the measurement of maximum locomotor capabilities. J. Zool. 258, 57-61 (2002).

34. Astley, H. C. \& Roberts, T. J. Evidence for a vertebrate catapult: elastic energy storage in the plantaris tendon during frog jumping. Biol. Lett. 8, 386-389 (2012).

35. Richards, C. \& Sawicki, G. Elastic recoil can either amplify or attenuate muscletendon power, depending on inertial versus fluid dynamic loading. J. Theor. Biol. 313, 68-78 (2012).

36. Warton, D. I., Wright, I. J., Falster, D. S. \& Westoby, M. Bivariate line-fitting methods for allometry. Biol. Rev. 81, 259-291 (2006).

\section{Acknowledgements}

This research was supported by the Rowland Junior Fellows Program at Harvard University.

\section{Author contributions}

C.J.C and C.R. performed the experiments and wrote the manuscript.

\section{Additional information}

Competing financial interests: The authors declare no competing financial interests.

Reprints and permission information is available online at http://npg.nature.com/ reprintsandpermissions/

How to cite this article: Clemente, C. J. and Richards, C. Muscle function and hydrodynamics limit power and speed in swimming frogs. Nat. Commun. 4:2737 doi: 10.1038/ncomms3737 (2013). 